\title{
EMBEDDINGS INTO GROUPS WITH ONLY A FEW DEFINING RELATIONS
}

\author{
Dedicated to the memory of Hanna Neumann \\ W. W. BOONE* and D. J. COLLINS
}

(Received 27 June 1972)

Communicated by M. F. Newman

It is a trivial consequence of Magnus' solution to the word problem for onerelator groups [9] and the existence of finitely presented groups with unsolvable word problem [4] that not every finitely presented group can be embedded in a one-relator group. We modify a construction of Aanderaa [1] to show that any finitely presented group can be embedded in a group with twenty-six defining relations. It then follows from the well-known theorem of Higman [7] that there is a fixed group with twenty-six defining relations in which every recursively presented group is embedded.

The results of the present paper are analogous for groups of the results of [2] about semigroups; however, no knowledge of [2] is required to read this paper.

1

Let $A$ be any finitely presented group. In view of the nature of our proposed theorem we may, without loss of generality, assume that $A$ has two-generatorssay $A=\left\langle a_{1}, a_{2}\right\rangle$. (See for example [8]).

We begin by regarding $A$ as a semigroup. In this role $A$ has four generators, namely $a_{1}, a_{2}, a_{1}^{-1}$ and $a_{2}^{-1}$. When convenient, we shall sometimes write $a_{3}$ and $a_{4}$ for $\mathrm{a}_{1}^{-1}$ and $a_{2}^{-1}$ respectively.

$$
\text { Let } A=\left(a_{1}, a_{2}, a_{3}, a_{4} ; R_{i}=1, i=1,2, \cdots, n\right) \text {. }
$$

We begin by applying to $A$ a construction of the type given on p. 307 of [4]. It will turn out that there is one delicate point in the argument. In order that this point will be clear, and to make the paper more easily readable, we specify the various constructions in detail.

The first author acknowledges support of the U. S. National Science Foundation. 
Our first step is to define the semigroup

$$
A_{*}=\left(a_{1}, a_{2}, a_{3}, a_{4}, p ; R_{i} p=p, a_{j} p=p a_{j} ; i=1,2, \cdots, j=1,2,3,4\right) .
$$

Next we give an alternative presentation of $A_{*}$. For this we write $a_{5}$ for $p$. Then by introducing additional generators and relations we can present $A *$ as

$$
A_{*}=\left(a_{1}, a_{2}, \cdots, a_{r} ; A_{j}=B_{j}, j=1,2, \cdots, s\right)
$$

where each relation is of the form $a_{\lambda}=a_{\mu} a_{v}, 1 \leqq \lambda, \mu, v \leqq r$. By suitable repetition, if necessary, we may assume $s=2^{t}$, for some $t$.

We now embed $A *$ in a semigroup $\mathfrak{L}$ on generators $\beta$ and $\gamma$. The embedding is defined by the mapping $\psi$ given by $a_{i}^{\psi} \equiv \beta \beta \gamma^{i} \beta \gamma^{r+1-i}$ and $(U V)^{\psi} \equiv U^{\psi} V^{\psi}$. Then $\mathfrak{L}=\left(\beta, \gamma ; A_{j}^{\psi}=B_{j}^{\psi}, j=1,2, \cdots, s\right)$.

Let $u=r+4$; then $A_{j}^{\psi}$ contains $u$ symbols and $B_{j}^{\psi}$ contains $2 u$ symbols. Writing $x_{i, j}$ for the $i$-th symbol of $A_{j}^{\psi}$ and $y_{i, j}$ for the $i$-th symbol of $B_{j}^{\psi}$ we define

and

$$
M \equiv x_{1,1} x_{1,2} \cdots x_{1, s} x_{2,1} \cdots x_{2, s} \cdots x_{u, 1} \cdots x_{u, s}
$$

$$
N \equiv y_{1,1} y_{1,2} \cdots y_{1, s} y_{2,1} \cdots y_{2, s} \cdots y_{2 u, 1} \cdots y_{2 u, s}
$$

(recall that $s=2^{t}$ ).

Let $\mathfrak{M}$ be the semigroup with presentation

$$
\begin{gathered}
\beta, \gamma, \varepsilon ; \\
\varepsilon \beta \beta=\beta \varepsilon \quad \varepsilon \gamma \beta=\beta \varepsilon \\
\varepsilon \beta \gamma=\gamma \varepsilon \quad \varepsilon \gamma \gamma=\gamma \varepsilon \\
M=N
\end{gathered}
$$

We now define the semigroup $\mathfrak{N}=\left(\alpha, \sigma ; \sigma \alpha \alpha=\alpha \alpha \sigma \alpha \alpha \sigma, \sigma \alpha \alpha=\alpha \alpha \sigma \sigma, M^{\mathfrak{}} N^{t}\right)$, where

$$
\beta^{\tau} \equiv \sigma \alpha, \gamma^{\tau} \equiv \sigma, \varepsilon^{\tau} \equiv \alpha \alpha \text { and }(Y Z)^{\tau} \equiv Y^{\tau} Z^{\tau} .
$$

We write $\chi$ for the composition of $\psi$ and $\tau$.

THEOREM 1. Let $U$ and $V$ be any two words of $A$. Then

(i) $(U V)^{x} \equiv U^{x} V^{x}$ (i.e. are identical as words);

(ii) If $U={ }_{A} V$ then $U^{x} p^{x} \sigma \alpha^{2 t}={ }_{n} V^{x} p^{x} \sigma \alpha^{2 t}$;

(iii) If $Z$ is a word of $\mathfrak{N}$ and $Z p^{x} \sigma \alpha^{2 t}={ }_{\mathfrak{n}} V^{x} p^{x} \sigma \alpha^{2 t}$, then there exists a word $W$ of $A$ such that $Z \equiv W^{*}$ and $W={ }_{A} V$.

Proof. Part (i) is trivial. Parts (ii) and (iii) are more or less in the literature. Initially one must note that where $U$ and $V$ are words of $U p={ }_{A *} V p$ if and only if $U={ }_{A} V$. Thereafter the most detailed source is probably [2]([10] contains only a sketch). 
[Unfortunately it has not been possible for us simply to quote results exactly as they occur in the literature. Also we have not been able to make our notation mesh precisely with that in the literature.]

We now employ the $G\left(\mathfrak{I}, \Phi_{0}\right)$ construction on page 307 of [5] with $\mathfrak{N}$ in the role of $\mathfrak{I}$. We rewrite $\mathfrak{N}$ as

and define

$$
\left(s_{1}, s_{2} ; P_{i}=Q_{i}, i=1,2,3\right)
$$

$$
\text { L } \mathfrak{N} *=\left(s_{1}, s_{2}, q ; P_{i} q=q Q_{i}, s_{j} q=q s_{j}, i=1,2,3, j=1,2\right) \text {. }
$$

For convenience we write the relations of $\mathfrak{R}$ * as $F_{i} q=q K_{i}, i=1,2, \cdots, 5$ and write $\Phi_{0} \equiv p^{\alpha} \sigma \alpha^{2 t} \equiv p^{*} s_{2} s_{1}^{2 t}$. Then the group $G=G\left(\mathfrak{N}, \Phi_{0}\right)$ has presentation:

$$
\begin{gathered}
s_{1}, s_{2}, q, t, k, a, d ; \\
a s_{j}=s_{j} a \quad d s_{j}=s_{j} d^{6} a d^{6} \\
d^{i} a d_{i} \bar{F}_{i} q=q K_{i} d^{i} a d^{i} \\
t a=a t \quad t d=d t \\
k a=a k \quad k d=d k \\
k\left(\Phi_{0}^{-1} q^{-1} t q \Phi_{0}\right)=\left(\Phi_{0}^{-1} q^{-1} t q \Phi_{0}\right) k
\end{gathered}
$$

where $i=1,2, \cdots 5, j=1,2$ and $\bar{F}_{i}$ is obtained from $F_{i}$ by replacing $s_{j}$ by $s_{j}^{-1}$.

For any word $W$ of $A$, let $W^{\theta}$ denote the word obtained from $W^{x}$ by replacing $s_{j}$ by $s_{j}^{-1}$.

THEOREM 2. For any word $W$ of $A, W={ }_{A} 1$ if and only if

$$
k^{-1}\left(\Phi_{0}^{-1} q^{-1}\left(W^{\theta}\right)^{-1} t W^{\theta} q \Phi_{0}\right) k={ }_{\mathrm{G}} \Phi_{0}^{-1} q^{-1}\left(W^{\theta}\right)^{-1} t W^{\theta} q \Phi_{0} \text {. }
$$

Proof. After sorting out the notation one sees that this is an immediate consequence of Theorem 1(ii) above and Lemma $\mathrm{C}$ and Technical Result (i) of [5].

At this point we begin to follow Aanderaa [1]. Let $k_{0} \equiv q \Phi_{0} k \Phi_{0}^{-1} q^{-1}$; then of course Theorem 2 says that $W={ }_{A} 1$ if and only if $k_{0}^{-1}\left(W^{\theta}\right)^{-1} t W^{\theta} k_{0}$ $={ }_{G}\left(W^{\theta}\right)^{-1} t W^{\theta}$. We shall sometimes regard $k_{0}$ as an abbreviation and at other times as a new generating symbol.

Let $C=\left\langle c_{1}, c_{2}\right\rangle$ be an isomorphic copy of $A$. It is worth stressing that we shall in fact embed $C$ rather than $A$.

Let $K_{1}=G * C$. We shall define a sequence of groups using the well-known $H N N$-construction (see [8] or [4]) or, to use alternative standard terminology in a 'word problem' context, Britton extensions. We shall call such a construction an $H N N B$-extension. It will not always be trivial to verify that we have a legitimate instance of the construction, i.e. that Britton's isomorphism condition holds. For the moment we simply give the presentations, reserving the verifications till later. They are: 


$$
\begin{aligned}
& K_{2}=\left(K_{1}, b_{1}, b_{2} ; b_{i}^{-1} s_{j} b_{i}=s_{j}, b_{i}^{-1} c_{j} b_{i}=c_{j}, b_{i}^{-1} k_{0} b_{i}=k_{0} c_{i}^{-1}, i, j=1,2\right) ; \\
& K_{3}=\left(K_{2}, f ; f^{-1}\left(a_{i}^{\varepsilon}\right)^{\theta} b_{i}^{\varepsilon} f=\left(a_{i}^{\varepsilon}\right)^{\theta}, f^{-1} k_{0} f=k_{0}, i=1,2 \varepsilon= \pm 1\right) ; \\
& K_{4}=\left(K_{3}, h ; h^{-1} t h=t f, h^{-1} k_{0} h=k_{0}, h^{-1} s_{j} h=s_{j}, j=1,2\right) .
\end{aligned}
$$

THEOREM 3. (i) $K_{1}$ is naturally embedded in $K_{4}$.

(ii) The defining relations of $C$ are consequences of the remaining thirty-three defining relations of $K_{4}$.

Before proving Theorem 3, we take the final step in our argument. It will be observed that $K_{4}$ has a number of commuting relations. Using a technique due essentially to Borisov [3] we can eliminate some of these.

THEOREM 4. $K_{4}$ can be embedded in a group $K_{4}^{\prime}$ with twenty-six defining relations

Proof. Define $a_{0} \equiv q \Phi_{0} a \Phi_{0}^{-1} q^{-1}$ and $d_{0} \equiv q \Phi_{0} d \Phi_{0}^{-1} q^{-1}$. Then we can present $K_{4}$ in such a way that the generator $k_{0}$ commutes with the generators $a_{0}, d_{0}$, $f, h$ and $t$. Also $b_{1}$ and $b_{2}$ commute with $s_{1}, s_{2}, c_{1}$ and $c_{2}$. If we twice apply Borisov's theorem in the form in which it appears in [6], it is clear that with our first application we can reduce the number of commuting relations by three, and with our second application by another four.

We now prove Theorem 3 , beginning with part (ii).

Let $W={ }_{A} 1$; we shall write $W_{b}$ and $W_{c}$ for the copies of $W$ in $b_{1}, b_{2}$ and $c_{1}$, $c_{2}$ respectively. We shall deduce $W_{c}=1$ from the relations of $K_{4}$ excluding the relations of $C$. We have, by Theorem 2

$$
k_{0}^{-1}\left(W^{0}\right)^{-1} t\left(W^{\theta}\right) k_{0}=\left(W^{\theta}\right)^{-1} t W^{\theta} .
$$

If we conjugate both sides by $h$ and use the relations involving $h$ we obtain

$$
k_{0}^{-1}\left(W^{\theta}\right)^{-1} t f W^{\theta} k_{0}=\left(W^{\theta}\right)^{-1} t f W^{\theta} .
$$

If we introduce suitable inverse pairs and use (*) we obtain

$$
k_{0}^{-1}\left(W^{\theta}\right)^{-1} f W^{\theta} k_{0}=\left(W^{\theta}\right)^{-1} f W^{\theta} \text {. }
$$

Now it follows from the definition of $\chi$ and $\theta$ that if

$$
W \equiv a_{i_{1}} a_{i_{2}} \cdots a_{i_{n}} \text { then } W^{\theta} \equiv a_{i_{1}}^{\theta} a_{i_{2}}^{\theta} \cdots a_{i_{n}}^{\theta} \text {. }
$$

Hence we may pass $f$ from left to right across $W^{\theta}$ to obtain $k_{0}^{-1} W_{b} k_{0}=W_{b}$. Since $k_{0}^{-1} b_{i} k_{0}=b_{i} c_{i}$, we have $W_{b} W_{c}=W_{b}$ and so $W_{c}=1$.

To prove part (i) we must establish three isomorphisms. 
(1) $K_{2}$ is an $H N N B$-extension of $K_{1}$. Here we must show that for each $i,\left\langle s_{j}, k_{0}, c_{i} ; j, l=1,2\right\rangle$ and $\left\langle s_{j}, k_{0} c_{i}^{-1}, c^{i} ; j, l=1,2\right\rangle$ are isomorphic under $s_{j} \rightarrow s_{j}$, $k_{0} \rightarrow k_{0} c^{-1}, c_{l} \rightarrow c_{l}$. This follows from the fact that $\left\langle s_{j}, k_{0}, c_{l}\right\rangle$ is the free product $\left\langle s_{j}, k_{0}\right\rangle^{*} C$ and $\left\langle s_{j}, k_{0}\right\rangle$ is free on these generators.

(2) $K_{3}$ is an $H N N B$-extension of $K_{2}$. This time we must look at $\left(a_{i}^{\varepsilon}\right)^{\theta} b_{i}^{\varepsilon} \rightarrow\left(a_{i}^{\varepsilon}\right)^{\theta}$, $k_{0} \rightarrow k_{0}$. We shall show that $\left\langle\left(a_{i}^{\varepsilon}\right)^{\theta}, k_{0}\right\rangle$ is free on these generators. Then when we map $K_{2} \rightarrow G$ by putting $b_{i}=1, i=1,2$ we map $\left\langle\left(a_{i}^{\varepsilon}\right)^{\theta} b_{i}^{\varepsilon}, k_{0}\right\rangle$ onto $\left\langle\left(a_{i}^{\varepsilon}\right)^{\theta}, k_{0}\right\rangle$. It suffices to prove that $\left\langle\left(a_{i}^{\varepsilon}\right)^{\theta}\right.$ is free in $\left\langle s_{1}, s_{2}\right\rangle$.

We recall the definition of $\theta$. Writing $a_{3}$ and $a_{4}$ for $a_{1}^{-1}$ and $a_{2}^{-1}$, we see that

$$
a_{i}^{\theta} \equiv\left(s_{2}^{-1} s_{1}^{-1}\right)^{2} s_{2}^{-(i+1)} s_{1}^{-1} s_{2}^{-(r+1-i)} .
$$

Let us call the right most $s_{1}^{-1}$ the central symbol of $a_{i}^{\theta}$. Then it is not hard to see that in a product $\left(a_{i}^{\theta}\right)^{\eta}\left(a_{j}^{\theta}\right)^{v}, \eta, v= \pm 1$ the central symbols of $a_{i}^{\theta}$ and $a_{j}^{\theta}$ are not cancelled unless $i=j$ and $\eta+v=0$. This extends to arbitrary products and the desired assertion follows.

(3) $K_{4}$ is an $H N N B$-extension of $K_{3}$. In this case we must prove that $D=\left\langle s_{j}, t, k_{0}\right\rangle$ and $E=\left\langle s_{j}, t f, k_{0}\right\rangle$ are naturally isomorphic. To do so we shall show that $D$ has presentation

$$
\left(s_{1}, s_{2}, k_{0} ; k_{0}^{-1}\left(W^{\theta}\right)^{-1} t W^{\theta} k_{0}=\left(W^{\theta}\right)^{-1} t W^{\theta}\right)
$$

where $W$ ranges over all relators of $A$.

Let $X$ be any word in $s_{1}, s_{2}, t$ and $k_{0}$, such that $X={ }_{G} 1$. We want to reduce $X$ to the empty word using only the displayed relations. If $X$ contains a subword $t^{\eta} W^{\theta} k$, where $W={ }_{A} 1$, we replace this subword by $W^{\theta} k^{v}\left(W^{\theta}\right)^{-1} t^{\eta} W^{\theta}$ and freely reduce the resulting word. This operation is clearly equivalent to an application of the given relations. Let $X_{1}$ be the result of iterating this procedure for as long as possible.

Suppose $X_{1}$ is non-empty. Since both $\left\langle s_{j}, t\right\rangle$ and $\left\langle s_{j}, k_{0}\right\rangle$ are free we may assume both $t$ and $k_{0}$ appear in $X_{1}$. We can apply Britton's Lemma [4] to $G$ with $k_{0}$ as stable letter. Hence $X_{1}$ contains a subword $Z_{1} t^{m_{1}} Z_{2} \cdots Z_{r} t^{m r} Z_{r+1}$ which belongs to $\left\langle a_{0}, d_{0}, t\right\rangle$. Next we may use $t$ as stable letter to deduce that there exist words $R_{1}$ and $R_{2}$ on $a$ and $b$ such that $Z_{r+1} q \Phi_{0}=R_{1} q \Phi_{0} R_{2}$ in the group $G_{2}$ of [5]. (For the moment we use the notation of [5].) This last equality yields the two equalities

$$
R_{1}^{-1} Z_{r+1} A_{i_{1}}^{\varepsilon_{1}} \cdots A_{i_{n}}^{\varepsilon_{n}}={ }_{G_{4}} 1 \text { and } \Phi_{0} R_{2} \Phi_{0}^{-1} B_{i_{1}}^{\varepsilon_{1}} \cdots B_{i_{n}}^{\varepsilon_{n}}={ }_{G_{4}} 1 .
$$

We require a lemma.

LEMMA. If $R Z A_{i_{1}}^{\varepsilon_{1}} \cdots A_{i_{n}}^{\varepsilon_{n}}={ }_{G_{4}} 1$, then $Z$ is a negative word on the $s_{j}$-symbols.

Proof. The $s$-reduced word obtainable from $A_{i_{1}}^{\varepsilon_{1}} \cdots A_{i_{n}}^{e_{n}}$ has form $\Pi_{k=1}^{n}\left(D_{m_{k}}^{i_{k}} a D_{m_{k}}^{i_{k}} \bar{\Delta}_{k}\right)^{\ell_{k}}$, by Lemma 14 of [5] (correcting a misprint). Moreover $m_{1}=0$ 
(for $\varepsilon_{1}=+1$ or $\varepsilon_{1}=-1$ ). It then follows from Lemma 12 of [5] that $Z$ cannot contain positive occurrences of $s_{j}$.

Picking up the main argument again we see that we now have exactly the hypotheses of Lemma 19 of [5] and thus $Z_{r+1} \equiv \bar{\Delta}$ where $\Delta q \Phi_{0}=g_{r_{*}} q \Phi_{0}$. In turn we obtain $\Delta \Phi_{0}={ }_{\mathfrak{n}} \Phi_{0}$. After sorting out the notation, we see that, by Theorem 1 (iii), $\Delta \equiv W^{x}$ for some relator $W$ of $A$. Thus $Z_{r+1} \equiv W^{\theta}$ and this is a contradiction.

We complete the whole argument by showing that $E$ has presentation

$$
\left(s_{1}, s_{2}, t_{1}, k_{0} ; \kappa_{0}^{-1}\left(W^{\theta}\right)^{-1} t_{1} W^{\theta} k_{0}=\left(W^{\theta}\right)^{-1} t_{1} W^{\theta}\right)
$$

where we write $t_{1} \equiv t f$ and $W$ ranges over all relators of $A$.Us ng the relations of $K_{3}$ we see that

$$
k_{0}^{-1}\left(W^{\theta}\right)^{-1} t_{1} W^{\theta} k_{0}={ }_{K_{3}} k_{0}^{-1}\left(W^{\theta}\right)^{-1} t W^{\theta} k_{0} W_{b} W_{c}
$$

and $\left(W^{\theta}\right)^{-1} t_{1} W^{\theta}=\mathbf{k}_{3}\left(W^{\theta}\right)^{-1} t W^{\theta} W_{b}$. So the displayed relations certainly hold in $E$. Using the fact that $k_{0}$ is a stable letter for $D$ over $\left\langle s_{j}, t\right\rangle$ we can then easily check that the natural map from $D$ to $E$ is an isomorphism.

One point in our construction is perhaps worth comment. Instead of Theorem 2 as stated, we could have asserted that $W={ }_{A} 1$ if and only if

$$
k^{-1}\left(\Phi_{0}^{-1}\left(W^{x}\right)^{-1} q^{-1} t q W^{x} \Phi_{0}\right) k={ }_{G} \Phi_{0}^{-1}\left(W^{x}\right)^{-1} q^{-1} t q W^{x} \Phi_{0} .
$$

Defining $t_{0} \equiv q^{-1} t q$ and $k_{0} \equiv \Phi_{0} k \Phi_{0}^{-1}$ we then have $W={ }_{A} 1$ if and only if $k_{0}^{-1}\left(W^{x}\right)^{-1} t_{0} W^{x} k_{0}={ }_{G}\left(W^{x}\right)^{-1} t_{0} W^{x}$. What is interesting is that if we attempt to repeat the construction with $t_{0}$ in the role of $t$ (and $\chi$ instead of $\theta$ ) the argument breaks down.

To see this we observe that there certainly exists a word $\Phi$ of $\mathfrak{N}$ such that $\Phi={ }_{\mathfrak{n}} \Phi_{0}$ and $Y \equiv \Phi \Phi_{0}^{-1}$ is freely reduced. Then it follows that

$$
k_{0}^{-1} Y^{-1} t_{0} Y k_{0}={ }_{G} Y^{-1} t_{0} Y \text { while } k_{0}^{-1} Y^{-1} t_{0} f Y k_{0} \neq{ }_{K_{3}} Y^{-1} t_{0} f Y \text {. }
$$

Thus $D$ and $E$ cannot be isomorphic in the natural way.

We conclude with a few speculative remarks concerning the best possible result along the lines of our theorem. It is of course tempting to conjecture that every finitely presented group is embeddable in a two-relator group. If this be so, then there exist two-relator groups with unsolvable word problem, - but the best result presently known for this question is that there exist groups with twelve relators and unsolvable word problem.

\section{References}

[1] S. Aanderaa, 'A proof of Higman's embedding theorem using Britton extensions of groups', Word Problems: decision problems and the Burnside problem in group theory 1-18. Studies in Logic and Foundations of Mathematics, (North-Holland Publ. Co., Amsterdam, 1973).

[2] W. W. Boone, D. J. Collins and Yu. V. Matijasevic, 'Embeddings into semigroups with only a few defining relations', Proc. of the Second Scandinavian Logic Symposium 27-40. Studies in Logic and Foundations of Mathematics, (North-Holland Publ. Co., Amsterdam, 1971). 
[3] V. V. Borisov, 'Simple examples of groups with unsolvable world problem', Math. Notes 6 (1969), 768-775, (Math. Zametki, 6 (1969), 521-532, Russian).

[4] J. L. Britton, 'The word problem', Ann. of Math. (1963), 16-32.

[5] D. J. Collins, 'Word and conjugacy problems in groups with only a few defining relations', Zeitschr. f. Math. Logik und Grundlagen d. Math. 15 (1969), 305-325.

[6] D. J. Collins, 'On a group embedding theorem of V. V. Borisov.' To appear in Bull. Lond. Math. Soc.

[7] G. Higman, 'Subgroups of finitely presented groups', Proc. Roy. Soc. Series A 262 (1961), 455-475.

[8] G. Higman, B. H. Neumann and H. Neumann, 'Embedding theorems for groups', J. London Math. Soc. 24 (1949), 247-256.

[9] W. Magnus, 'Das Identitätsproblem für Gruppen mit einer definierenden Relation,' Math. Ann. 106 (1932), 295-307. (See also W. Magnus, A. Karass and D. Solitar, Combinatorial Group Theory, Interscience 1966, 252-278.)

[10] Yu. V. Matijasevic, 'Simple examples of undecidable associative calculi', Soviet Math. 8 (1967), 555-557.

University of Illinois at Urbana-Champaign U.S.A.

Queen Mary College, University of London

England 